\title{
Improvement of a Figure Copying Deficit during Sub-Sensory \\ Galvanic Vestibular Stimulation
}

David Wilkinson $^{1}$, Olga Zubko ${ }^{1}$, Joseph DeGutis ${ }^{2,3}$, William Milberg ${ }^{2,3}$ \& Jonathan Potter ${ }^{4}$

${ }^{1}$ Department of Psychology, University of Kent, U.K.

${ }^{2}$ Geriatric Neuropsychology Laboratory, New England Geriatric, Research, Education \& Clinical Center, Veterans Affairs Boston Healthcare System, U.S.A.

${ }^{3}$ Department of Psychiatry, Harvard Medical School, U.S.A.

${ }^{4}$ Healthcare of Older People Department, Kent \& Canterbury Hospital, East Kent Hospitals University NHS Foundation Trust, UK.

Correspondence: David Wilkinson, Ph.D., Department of Psychology, University of Kent, Canterbury, Kent, CT2 7NP, UK. Email: dtw@kent.ac.uk. Phone: +44 (0) 1227824772. Fax: +44 (0) 1227827030.

Acknowledgments: We thank patient A.A. and his wife for their patient assistance during testing, and are grateful to the Medical Reprographics Unit at the Kent and Canterbury Hospital for their help in preparing the CT images for presentation. 
Improvement of a Figure Copying Deficit during Sub-Sensory

Galvanic Vestibular Stimulation 
We describe the effects of galvanic vestibular stimulation (GVS) on an individual who, following right hemisphere stroke, is unable to copy figures accurately. His copies contain most of the constituent elements, but are poorly integrated and drawn in a seemingly haphazard manner. To test whether GVS could help overcome these difficulties, we administered the Rey-Osterrieth Complex Figure Copy task while manipulating both the presence and laterality of the galvanic signal. The signal was applied at a level that was too low to elicit sensation which ensured that the individual was unaware of either when or on what side he was being stimulated. Relative to a sham condition, two consecutive blocks of GVS increased both the accuracy with which the main configural elements of the figure were reconstructed, and there was some, albeit less consistent evidence, that these were drawn in a more wholistic as opposed to piecemeal manner. Improvement was not reliant on the polarity of the stimulating electrodes. These results suggest that GVS can help overcome difficulties in the perception and/or reconstruction of hierarchical visual form, and thereby uncover a new link between vestibular information processing and visual task performance. 


\section{Introduction}

Galvanic vestibular stimulation (GVS) is a non-invasive procedure involving the delivery of electrical current to the part of the scalp that overlies the vestibular nerves (Coats, 1972). These nerves convey signals from the balance organs of the inner ear to brainstem nuclei, which indirectly project fibres to temporalparietal cortex which is associated with the construction and maintenance of multi-sensory spatial representations (Guldin \& Grüsser, 1996; Kerkhoff, 2003). The need for vestibular information in forming these representations is aptly demonstrated by the unusually high incidence of visuo-spatial impairment in patients who suffer from peripheral vestibular disease (Schautzer, Hamilton, Kalla, Strupp, \& Brandt, 2003).

Given this link between the vestibular and visual systems, it is perhaps unsurprising that experimental alterations to the vestibular signal affect visual perception. It has long been known that caloric vestibular stimulation, a procedure in which the vestibular nerves are activated by injecting cold water into the external ear canal, can reduce contralesional inattention in patients suffering from hemi-spatial neglect (Cappa, Sterzi, Vallar, \& Bisiach, 1987). In keeping with this finding, Rorsman, Magnusson, \& Johansson (1999) showed that GVS can spontaneously reduce the number of left-sided omissions in the line crossing test (Albert, 1973), a notable finding because unlike caloric stimulation, GVS does not invoke unpleasant sideeffects and is quick and simple to administer. Building on this result, Saj, Honoré \& Rousseaux (2006) showed that low-level GVS can reduce contralesional deviation of the subjective vertical in patients suffering from neglect. Along separate lines, Wilkinson, Ko, Kilduff, McGlinchey, \& Milberg (2005) showed that the face matching skills of a prosopagnosic patient could be significantly improved during GVS. This finding was particularly significant because it suggested that GVS can remediate visual deficits other than contralesional inattention. Nevertheless, the matching task employed by Wilkinson and colleagues could be accurately performed by detecting changes in superficial image properties related to luminance and contrast, rather than in the identity and arrangement of visual features. As a consequence, only limited conclusions could be drawn about the types of visual task in which GVS moderates performance. 
In the present study, we describe the effects of GVS in a brain-damaged patient who, in the absence of primary sensory or motor loss, cannot copy figures accurately. His copies are characterised by a failure to correctly size and arrange the individual component parts, and by a tendency to complete the main structural elements in a piecemeal manner. Problems of this nature can arise following damage to either the left or right cerebral hemisphere, and are often taken to reflect a perceptual failure to integrate local and global form and/or executive difficulties in organisation and planning (Lezak, 1983). To assess the effects of GVS on the patient's copying deficit, we employed the Rey Osterrieth Complex Figure (ROCF) copy test (Osterrieth, 1944; Rey, 1941). Compared to the matching and line crossing/vertical orientation tasks described above, this involves the apprehension of a more detailed hierarchical visual form and subsequent co-ordination of a relatively complex sensori-motor response. Given that so little is known about the range of visual tasks that are affected by GVS in brain-damaged patients, we reasoned that it would be instructive to show whether GVS can enhance performance in a higher-level task of this nature.

\section{Method}

Prior to testing, a favourable ethical opinion was obtained from the East Kent Local Research Ethics Committee.

\section{Case presentation}

Patient A.A is a right-handed male born in 1931. He was briefly admitted to an acute stroke ward in 2005 following the sudden onset of slurred speech, left facial weakness and no power in the left upper limb. His visual fields were intact to confrontation but sensory inattention was present in the left upper and lower limbs. A head CT scan carried out soon after admission showed a lesion within the territory of the right middle cerebral artery (see Figure 1).

Figure 1 about here 
The patient was referred to us some three years after his stroke on the basis of the hemi-spatial neglect shown during initial clinical presentation. Formal tests of line bisection, symbol cancellation and visual feature conjunction search showed no residual spatial bias, but the patient did show left-sided visual extinction when presented with computerised stimuli in left and right fields simultaneously. More remarkable was his failure to accurately copy and arrange the elements of line drawings, regardless of whether these were located on the right or left side of the drawings (see Figure 2). Further assessment of his visual perception using the Birmingham Object Recognition Battery (Riddoch \& Humphreys, 1993) showed normal performance on all sub-tests of perceptual matching, viewpoint invariance, object naming and semantic matching, although abnormal performance was noted during (1) drawing from memory, which was laboured and spatially distorted, and (2) the overlapping figures test, which produced unusually slow, albeit accurate, naming of stimuli when these spatially overlapped compared to when they were presented side by side. Despite this difficulty in perceptual segmentation, the patient was able to name singularly presented objects without hesitation and did not report difficulty recognising objects in everyday life, a view shared by his wife.

Figure 2 about here

\section{Procedure}

To establish whether the patient's copying deficit could be temporarily improved by GVS, the ROCF task was administered over a number of sessions while the patient received either active or sham stimulation. Some of the sessions comprised two separate blocks of stimulation, and on such occasions a separate copy of the ROCF was drawn each time. The participant's signed informed consent was obtained at the start of each test session, after which the ROCF was positioned along the patient's mid-sagittal plane with a blank response sheet placed directly below. The patient was instructed to copy the figure, in his own time, as carefully and accurately as possible. The examiner sat opposite and on a separate piece of paper tracked the 
order in which the patient constructed the stimulus. Different blocks were separated by a 5minute rest period, and different sessions were separated by two weeks.

Stimulation was started a minute before the patient was instructed to pick up the pencil to begin the task and stopped as soon as he put down the pencil to indicate that he had finished. Copies typically took approximately five minutes to complete although we did not measure the precise duration. During active stimulation, bipolar, binaural direct current was applied to the left and right mastoids via $3 \mathrm{~cm}^{2}$ carbon rubber electrodes coated with electro-conductive gel. The left/right polarity of the current was varied across sessions and blocks (see below), and the signal was convolved with a random noise component that had a frequency of $1 \mathrm{kHz}$, followed a Gaussian distribution, and was set at $0.25 \sigma$ of mean signal intensity. Under sham stimulation, the patient wore the electrodes and given that the electrical current was delivered at $90 \%$ of sensory threshold (threshold $=1.1 \mathrm{~mA}$ across all sessions), he was unable to differentiate sham from active stimulation (see Wilkinson, Nicholls, Pattenden, Kilduff, \& Milberg, 2008 for a description of the stair-case procedure used to find threshold). Direct current was delivered from a laptop PC to a stimulus isolation unit (A-M Systems 2200 analogue stimulus isolator) using National Instruments LabVIEW 6.0 and a dual output Microstar D/A board. The apparatus remained in full view of the participant throughout both active and sham stimulation.

\section{Results}

The copies were scored blindly using the comprehensive scoring method of the Boston Qualitative Scoring System (BQSS) (Stern et al., 1999). This method involves measurement of how well the three main hierarchical levels of the figure (configural elements, clusters and local details) are reproduced. Each level is assessed for both its presence and accuracy, the latter of which is based on a number of variables including completeness, size, proportion, and angle correctness. Together these measures comprise a summary statistic known as the Copy Presence Accuracy (CPA) score which is taken as a global measure of visual perception / visual reconstruction. In addition to the CPA, the BQSS produces another summary score, Organization. This measure is derived from the separate measures of Fragmentation and Planning which 
together quantify the order in which the individual elements are drawn and whether elements that can be perceptually grouped are drawn as single units. Unlike the CPA score, the Organization score therefore reflects the way in which the figure is put together as opposed to its final appearance.

These summary scores can be interpreted with reference to the frequency distributions provided by a normally-distributed, matched control sample. The sample comprises 56 neurologically healthy, gender- and age- (i.e. 70-79years) matched individuals from North America who are predominantly Caucasian, speak English as a first language and have a mean education of 13.8 years. To assist comparisons, the scores of the control sample are converted into $\mathrm{T}$ scores which are standardised to have a mean of 50 and standard deviation of 10. The interpretation of any given $\mathrm{T}$ score is therefore based on its deviation from the standardisation sample mean of 50. Summary scores are scaled such that higher $\mathrm{T}$ scores reflect better performance, and to further assist interpretation, cumulative percentile equivalents are assigned to each $\mathrm{T}$ score (see Table 1 Legend). The following clinical guidelines are recommended when interpreting a patient's T score: $<20=$ severely impaired, 20-29=moderately to severely impaired, 30-39=mildly impaired, 40-59=average, $60-69=$ above average, $\geq 70=$ superior.

In the following section, we separately present the effects of GVS on the patient's CPA and Organisation summary scores.

\section{CPA scores}

In session 1, patient A.A. was administered sham stimulation and produced a copy that, based on its corresponding $\mathrm{T}$ score of 36, fell into the mildly impaired range (see Figures 3 and 4 and Table 1). In session 2, left anodal/right cathodal stimulation was associated with more accurate performance that fell just inside the average range $(\mathrm{T}=41)$. In session 3 , the polarity of stimulation was reversed across two consecutive blocks of stimulation to test whether yet greater improvement could be elicited; this same reversal induced significant improvement in the face matching skills of prosopagnosic patient R.C. (see Wilkinson et al., 2005). In block 1, the patient's score fell in the average range $(\mathrm{T}=51)$ when stimulated with left anodal/right cathodal current, but in block 2 moved to the above-average range $(T=60)$ when he 
was stimulated with right anodal/left cathodal current. In session 4 we tested whether improvement still occurred if polarity was reversed in the opposite direction. In block 1 his performance fell just inside the lower limit of average during right anodal/left cathodal current $(\mathrm{T}=41)$, but again increased to aboveaverage during a subsequent block of left anodal/right cathodal current $(T=60)$. In session 5 we tested whether it actually mattered if electrode polarity was reversed across blocks or whether two blocks of the same polarity were sufficient to elevate performance. In line with the latter, two blocks of left anodal/right cathodal current moved his score from mildly impaired $(\mathrm{T}=32)$ in block 1 to average $(\mathrm{T}=55)$ in block 2 . In two final sessions, we tested whether performance naturally improved across two blocks of trials, regardless of stimulation. In Session 6, sham stimulation was applied in two consecutive blocks but led to no improvement (block1, $\mathrm{T}=46$; block 2, $\mathrm{T}=46$ ). The same pattern was observed across two blocks of sham in Session 7 (block 1, $\mathrm{T}=41$; block 2, $\mathrm{T}=41$ ).

\section{Organisation scores}

As can be seen from Table 1 and Figure 4, GVS affected the Organization summary score in a less consistent manner than the CPA score. The initial session of sham stimulation produced a $\mathrm{T}$ score $<20$ that fell within the severely impaired range. In the subsequent session of left anodal/right cathodal stimulation his score moved to 'average' $(\mathrm{T}=45)$. In session 3 , his performance did not move outside the average range across one block of left anodal/right cathodal stimulation and a subsequent block of right anodal/left cathodal stimulation, though the corresponding $\mathrm{T}$ score did show upward movement (block $1, \mathrm{~T}=45$; block 2, $\mathrm{T}=53$ ). In block 1 of session 4 , right anodal/left cathodal stimulation was associated with mildly impaired performance $(\mathrm{T}=30)$ which rose to above-average in block 2 during left anodal/right cathodal stimulation $(\mathrm{T}=86)$. In Session 5, performance remained within the average range across two blocks of left anodal/right cathodal stimulation $(T=45)$. In Sessions 6 and 7 , performance again remained unchanged across two blocks of sham stimulation $(\mathrm{T}=45)$. 
To address the fact that it could only take a small change in the patient's $\mathrm{T}$ score to move up a category (which could in turn lead to an over-estimation of the actual level of improvement), we constructed $95 \%$ confidence intervals from two standard errors of the control data and placed these around the two summary scores from block 1 of each of the stimulation sessions (sessions 3, 4 and 5). We then examined whether the mean score obtained in block 2 of each of these sessions exceeded the upper confidence limit. This was found to be the case in all cases (except for the Organisation score in Session 5), indicating that the improvement was unlikely to reflect normal variation of the block 1 mean.

Figures 3 and 4 and Table 1 about here

\section{Discussion}

The data indicate that two blocks of sub-sensory GVS improved patient A.A's copying performance relative to the sham condition; only active stimulation led to an improvement in the patient's CPA score within each session, and only active stimulation elevated his CPA score to above-average. Table 1 indicates that much of the improvement stemmed from more accurate reproductions of the main configural elements of the figure, though there was also some, albeit less consistent, evidence from the Organisational summary score that the component parts were assembled in a more systematic manner. These results indicate that the types of visual task in which beneficial effects can be found are broader than hitherto believed. Previous reports have shown that GVS can enhance both the cancellation of simple line stimuli presented in the contralesional field (Rorsman et al., 1999) and the matching of superficial image properties in non-neglected space (Wilkinson et al., 2005). Here we show a facilitatory effect in a patient who struggles to produce integrated copies of a relatively complex, hierarchical visual form.

Despite the consistent influence of two consecutive stimulation blocks on copying performance, we should point out that other aspects of performance were quite variable. Across the study as a whole, the initial block of active stimulation generated summary scores that varied from moderate/severely impaired to the lower end of average. There was also considerable performance variation between the first and last sham sessions which we presume could reflect carry-over from previous stimulations or practice, as opposed to 
mere random error. Inter-sessional variability is relatively common in longitudinal studies of brain-damaged individuals, and the opposing effects of practice and treatment carry-over on one hand, and fatigue and treatment habituation on the other, will need to be unpicked if the underlying magnitude of effect is to be accurately estimated. For the time being, we are encouraged by the fact that the beneficial effects of stimulation survive these unpredictable baseline shifts.

A key question now is to determine the underlying visual processes that are affected by vestibular stimulation. Studies of neglect patients suggest that the effects of vestibular stimulation are sufficiently selective as to rule-out non-specific arousal, and rather result from the modulation of multi-sensory, egocentric, spatial representations which in neglect are pathologically shifted towards ipsilesional space (see Magnusson, Pyykkö, \& Jäntti, 1985; Vallar, Papagno, Rusconi, \& Bisiach, 1995). By boosting sensory inputs from the neglected side, vestibular stimulation is thought to add an opposing spatial bias that somehow counteracts this underlying distortion (see Kerkhoff, 2003). In the present case, the beneficial effects of GVS on figure copying cannot be easily attributed to such a lateralised effect, thereby implying that asymmetrical changes to the left and right vestibular signal affect vision in a way other than simply reweighting the corresponding sides of perceptual space. Indeed, functional brain imaging studies show that GVS increases blood flow across bilateral peri-sylvia which is associated with a variety of visual processes (Bense, Thomas, Yousry, Brandt, \& Dieterich, 2001; Dieterich et al., 2003; Fink et al., 2003), which perhaps explains why other experiments have shown improvements in mental imagery and face matching. It is, however, important to point out that vestibular stimulation has been associated with transient recovery from a number of non-visual disorders including aphasia, hemiplegia, central pain and mania (Dodson, 2004; McGeoch, Williams, Lee \& Ramachandran, 2008; Miller \& Ngo, 2007; Schiff \& Pulver, 1999; Yamamoto, Struzik, Soma, Ohashi, \& Kwak, 2005). This widespread influence may point to the engagement of an additional, more generic compensatory response during stimulation. For example, Shiff and Pulver (1999) propose that vestibular stimulation affects the gating of cortico-cortico processing in thalamic nuclei, which in turn facilitates the temporary re-integration of damaged cortical regions (Schiff \& Pulver, 1999). A major challenge will be to establish the extent to which the visual recovery brought about 
by changes in the vestibular signal results from the modulation of specific processes within the vestibularvisual pathway (as is believed to be the case in hemi-spatial neglect) as opposed to the engagement of more global, compensatory processes that mediate cortical processing across a more multi-functional scale.

From a methodological standpoint, the data raise interesting questions about the best stimulation protocol to administer. The patient's copying ability was most enhanced by two blocks, as opposed to a single block, of stimulation, which implies that 'more is better'. At this time we cannot infer whether it is more time or more repeated blocks that are important as findings from transcranial direct current stimulation (tDCs), a similar procedure, suggest that both factors facilitate cognitive performance (Boggio et al., 2007; Ohn et al., 2008). An added complication is that given the close temporal interval (approx. 5mins) between the two blocks of stimulation, the improvement seen in the second block may have partly reflected an aftereffect from the prior block. This suggestion is based on data from tDCs which indicates that 5 minutes of tDCs applied at $1 \mathrm{~mA}$ can produce motor after-effects that last up to 10minutes (Nitsche \& Paulus, 2000). But what is impressive about the present study is that substantial improvement occurred only 10 minutes after stimulation onset (i.e. the time taken for the patient to draw the figure, take a break, and then re-draw for a second time). This leaves much room to explore the possibility that stronger, more enduring gains will be achieved with longer or more repetitive protocols.

A second notable aspect of the protocol was that patient A.A.'s improvement was not dependent on the relative positions of the two electrodes. That is, it did not matter if the anode was placed contralesionally across two stimulation blocks or whether polarity was switched from left/right to right/left. This configuration contrasts with the left anodal/right cathodal configuration needed to remediate the lateralised symptoms of hemi-spatial neglect. The reason for this discrepancy is unclear though some insight may again be gained from the fMRI studies cited above which show that although regions in both cerebral hemispheres are activated during GVS regardless of which side the anode is placed, activation is slightly greater on the cathodal side. In the present case, this bilateral pattern of activation may have been important because the coding and subsequent integration of local and global form is known to draw on processes in both cerebral hemispheres (see Delis, Robertson, \& Efron, 1986). In the case of neglect, however, correct placement of 
the anode may be needed to ensure that the resulting asymmetry in hemispheric activation runs counter to the contralesional spatial bias. Clearly, more study is needed to determine how changes to both the frequency and polarity of stimulation constrain behavioural outcome.

In sum, the remediative effect reported here adds to an emerging consensus that a range of visual cognitive impairments can respond favourably to GVS. Historically, GVS has been used as a tool to investigate how vestibular information mediates the autonomic control of balance and movement (see Fitzpatrick and Day, 2004). The results of the current study provide further evidence that higher-level processes associated with perception and attention are also affected (see Hanes and McColum, 2006). In rehabilitative terms, the procedure holds particular appeal over other forms of sensory remediation such as contralesional limb movement and neck muscle vibration because it appears to affect a broader range of task impairments and is less labour intensive. Such optimism must however be tempered by the fact that only a few GVS studies have been conducted, none of which have involved large sample sizes, random controlled procedures, dose-ranging manipulations, or detailed assessments of mechanism of effect. While our findings give reason to further investigate the therapeutic value of GVS in neuropsychological patients, we are wary of over-determining their significance at this preliminary stage. 
Albert, M. (1973). A simple test of visual neglect. Neurology, 23, 658-664.

Bense, S., Thomas, S., Yousry, T., Brandt, T., \& Dieterich, M. (2001). Multisensory cortical signal increases and decreases during vestibular galvanic stimulation (fMRI). Journal of Neurophysiology, 85, 886-899.

Boggio, P., Nunes, A., Rigonatti, S., Nitsche, M., Pascual-Leone, A., \& Fregni, F. (2007). Repeated sessions of noninvasive brain DC stimulation is associated with motor function improvement in stoke patients. Restorative Neurology and Neuroscience, 25, 123-129.

Cappa, S., Sterzi, R., Vallar, G., \& Bisiach, E. (1987). Remission of hemineglect and anosognosia during vestibular stimulation. Neuropsychologia, 25, 775-782.

Coats, A. C. (1972). Limit of normal of the galvanic body-sway test. The Annals of Otology, Rhinology and Laryngology, 81, 410-416.

Delis, D. C., Robertson, L. C., \& Efron, R. (1986). Hemispheric specialization of memory for visual hierarchical stimuli. Neuropsychologia, 24, 205-214

Dieterich, M., Bense, S., Lutz, S., Drzezga, A., Stephan, T., Bartenstein, P., \& Brandt, T. (2003).

Dominance for vestibular cortical function in the non-dominant hemisphere. Cerebral Cortex, 13, 994-1007.

Dodson, M. (2004). Vestibular stimulation in mania: a case report. Journal of Neurology, Neurosurgery \& Psychiatry, 75, 168-169. 
Fink, G. R., Marshall, J. C., Weiss, P. H., Stephan, T., Grefkes, C., Shah, N. J., Zilles, K., \& Dieterich, M. (2003). Performing allocentric visuospatial judgments with induced distortion of the egocentric reference frame: An fMRI study with clinical implications. Neuroimage, 20, 1505-1517.

Fitzpatrick, R., \& Day, B. (2004). Probing the human vestibular system with galvanic vestibular stimulation. Journal of Applied Physiology, 96, 2301-2316.

Guldin, W., \& Grüsser, O. J. The anatomy of the vestibular cortices of primates. In: Le Cortex Vestibulaire Editions IRVNN, edited by Collard M, Jeannerod M, and Christen, Y. Paris: Ipsen 1996, 17-26.

Hanes, D., \& McCollum, G. (2006). Cognitive-vestibular interactions: A review of patient difficulties and possible mechanisms. Journal of Vestibular Research, 16, 75-1.

Kerkhoff, G. (2003). Modulation and rehabilitation of spatial neglect by sensory stimulation. Progress in Brain Research, 142, 257-271.

Lezak, M. (1983). Neuropsychological Assessment. Oxford: OUP.

McGeoch, P., Williams, L., Lee, R., \& Ramachandran, V. (2008). Behavioural evidence for vestibular stimulation as a treatment for central post-stroke pain. Journal of Neurology, Neurosurgery \& Psychiatry, $79,1298-1301$

Magnusson, M., Pyykkö I., \& Jäntti, V. (1985). Effect of alertness and visual attention on optokinetic nystagmus in humans. American Journal of Otolaryngology, 6, 419-425. 
Miller, S., \& Ngo, T. (2007). Studies of caloric vestibular stimulation: implication for the cognitive neurosciences, the clinical neurosciences and neurophilosophy. Acta Neuropsychologica, 19, 183-203.

Nitsche, M., \& Paulus, W. (2000).Excitability changed induced in the human motor cortex by weak transcranial direct current stimulation. Journal of Physiology 527, 633-639.

Ohn, S., Park, C., Yoo, W., Ko, M., Choi, K., Kim, G., Lee., Y., \& Kim, Y. (2008). Time-dependent effect of trans-cranial direct current stimulation on the enhancement of working memory. NeuroImage, 19, 43-47.

Osterrieth, P. (1944). Le test de copie d'une figure complexe. Contribution a l'etude de la perception et de la memoire. Archives de Psychologie, 30, 206-256.

Rey, A. (1941). L'examen psychologique dans les cas d'encephalopathie traumatique. Archives de Psychologie, 30, 206-256.

Riddoch, M. J., \& Humphreys, G. W. (1993). BORB: The Birmingham Object Recognition Battery. Hove, Sussex: Lawrence Erlbaum.

Rorsman, I., Magnusson, M., \& Johansson, B. B. (1999). Reduction of visuo-spatial neglect with vestibular galvanic stimulation. Scandinavian Journal of Rehabilitation Medicine, 31, 117-124.

Saj, A., Honoré, J., \& Rousseaux, M. (2006). Perception of the vertical in patients with right hemispheric lesion: effect of galvanic vestibular stimulation. Neuropsychologia, 44, 1509-1512.

Schautzer, F., Hamilton, D., Kalla, R., Strupp, M., \& Brandt, T. (2003). Spatial memory deficits in patients with chronic bilateral vestibular failure. Annals of The New York Academy of Science, 1004, 316-24. 
Shiff N., \& Pulver, M. (1999). Does vestibular stimulation activate thalamocortical mechanisms that reintegrate impaired cortical regions? Proceedings of the Royal Society, London B Series, 266, 421-423.

Stern, R., Javorsky, D., Singer, E., Singer-Harris, N., Somerville, J., Duke, L., Thompson, J., \& Kaplan, E. (1999). The Boston Qualitative Scoring System. Florida: Psychological Assessment Resources.

Vallar, G., Papagno, C., Rusconi, M., \& Bisiach, E. (1995). Vestibular stimulation, spatial hemineglect and dysphasia, selective effects. Cortex, 31, 589-93.

Wilkinson, D., Ko, P., Kilduff, P., McGlinchey, R, \& Milberg, W. (2005). Improvement of a face perception deficit via subsensory galvanic vestibular stimulation. Journal of the International Neuropsychological Society, 11, 925-929.

Wilkinson D., Nicholls, S., Pattenden, C., Kilduff, P., \& Milberg, P. (2008). Galvanic vestibular stimulation speeds visual memory recall. Experimental Brain Research, 189, 243-8.

Yamamoto, Y., Struzik, Z., Soma, R., Ohashi, K., \& Kwak, S. (2005). Noisy vestibular stimulation improves autonomic and motor responsiveness in central neurodegenerative disorders. Annals of Neurology, $58,175-81$. 


\section{Figure Legends}

Figure 1. Computerised tomography scan of patient A.A's brain lesion. The slices indicate a large area of low attenuation affecting the right fronto-parieto region, extending from the level of the superior cerebral hemisphere down to the level of the sphenoid ridge. There was minor involvement of the anterior aspect of the right temporal lobe. No other focal abnormalities were present, and there was no haemorrhage or mass effect. $\mathrm{L}=$ left, $\mathrm{R}=$ right.

Figure 2. Patient A.A.s' figure copy performance during initial screening. The top three templates are reproduced from the Birmingham Object Recognition Battery (Riddoch and Humphreys, 1993), and the bottom template depicts the Rey-Osterrieth Complex Figure (see Osterrieth, 1944).

Figure 3. Patient A.A.'s reproductions of the Rey-Osterrieth Complex Figure during stimulation. AL = anode left/cathode right; $\mathrm{AR}=$ anode right/cathode left; NS = no stimulation (sham).

Figure 4. CPA and Organisational summary $\mathrm{T}$ scores expressed as a cumulative percentage of the standardised control sample. Sess $=$ Session, $\mathrm{AL}=$ anode left/cathode right; $\mathrm{AR}=$ anode right/cathode left; $\mathrm{NS}=$ no stimulation (sham). Dashed lines indicate cut-offs for the clinical categories identified by the Boston Qualitative Scoring System. Please note that the separate categories of 'moderately to severely impaired' ( $<2$ nd percentile) and 'severely impaired' $\left(<1^{\text {st }}\right.$ percentile) are grouped together. 


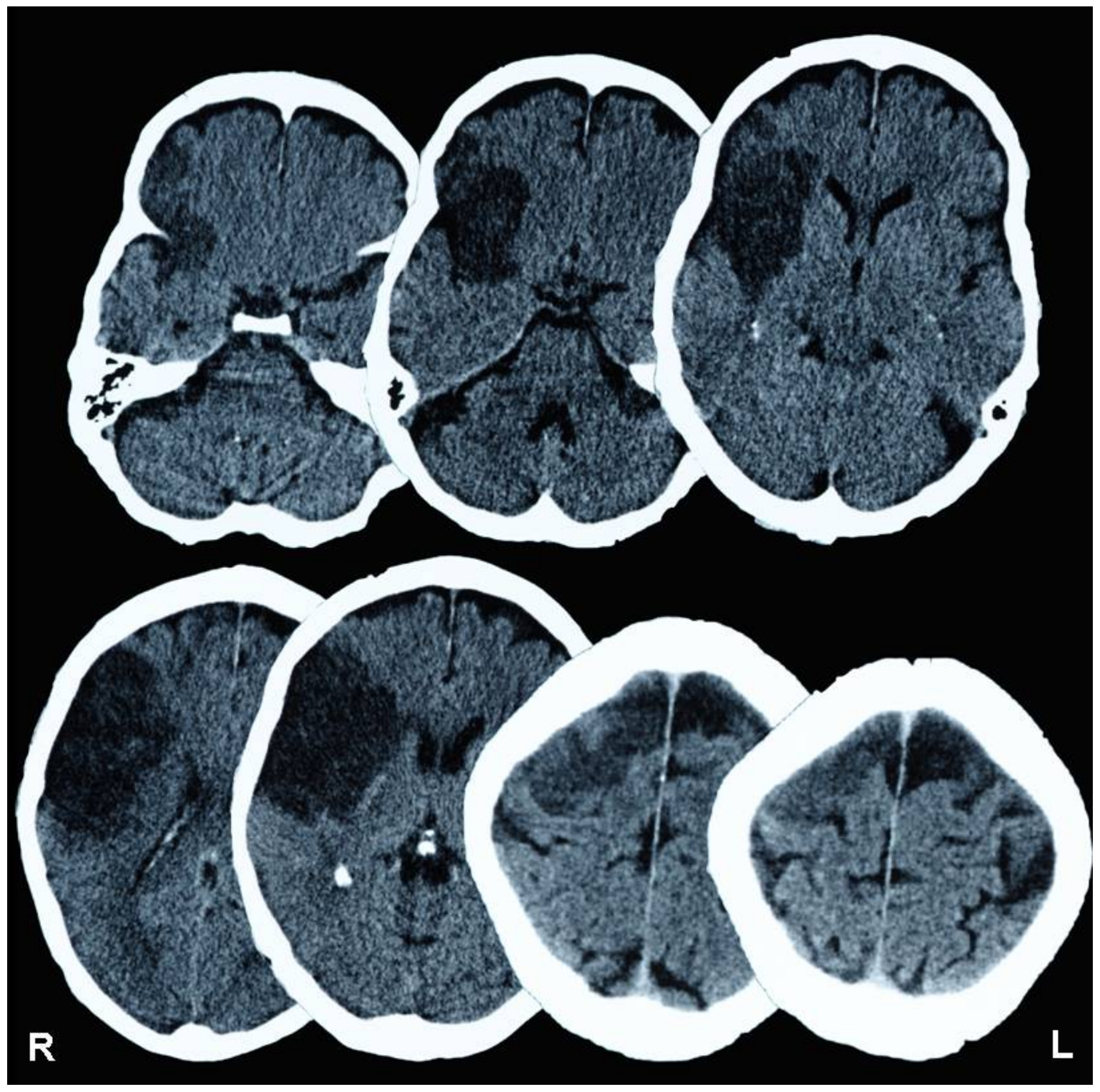

Figure 1 

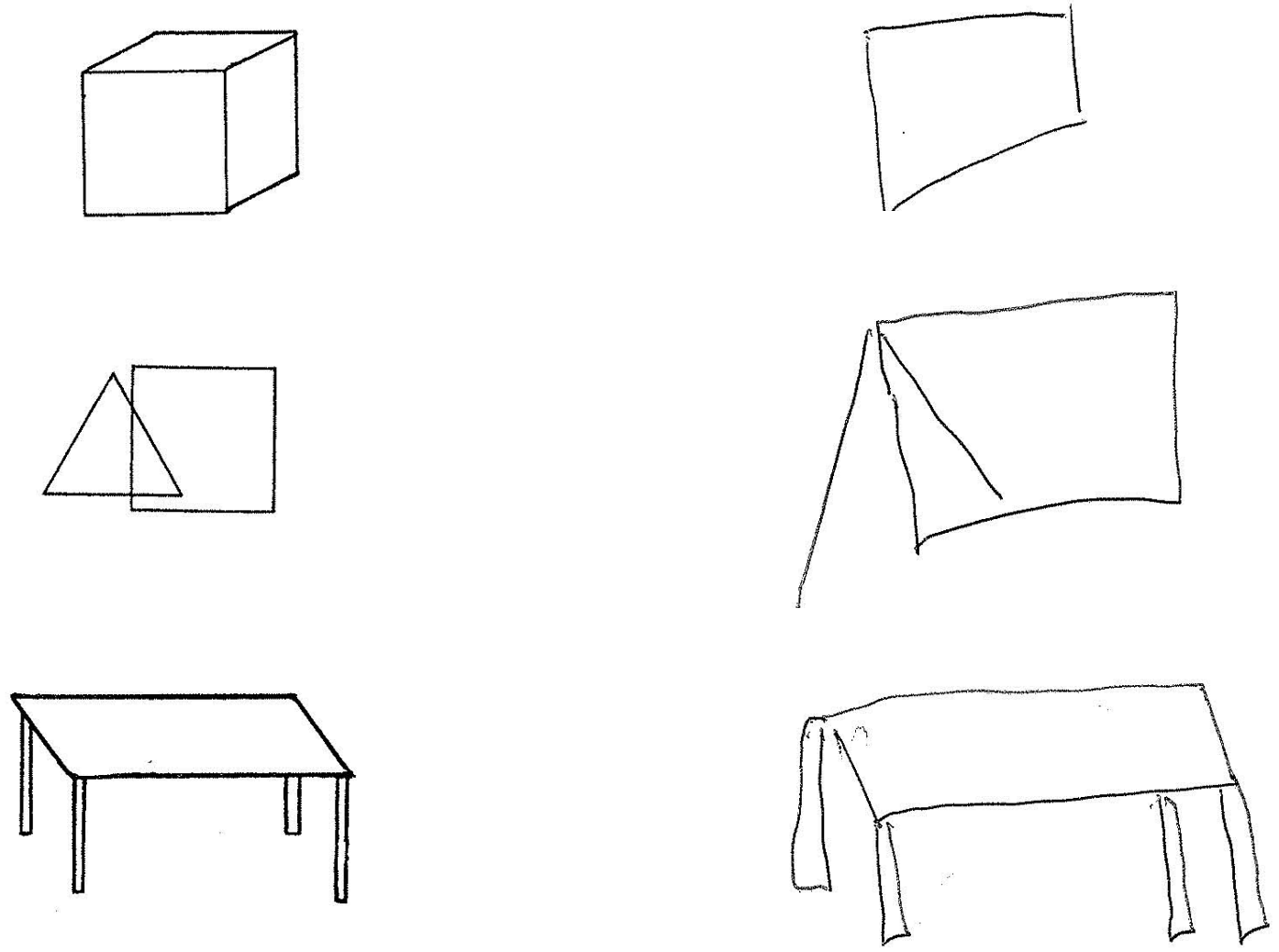

Figure 2
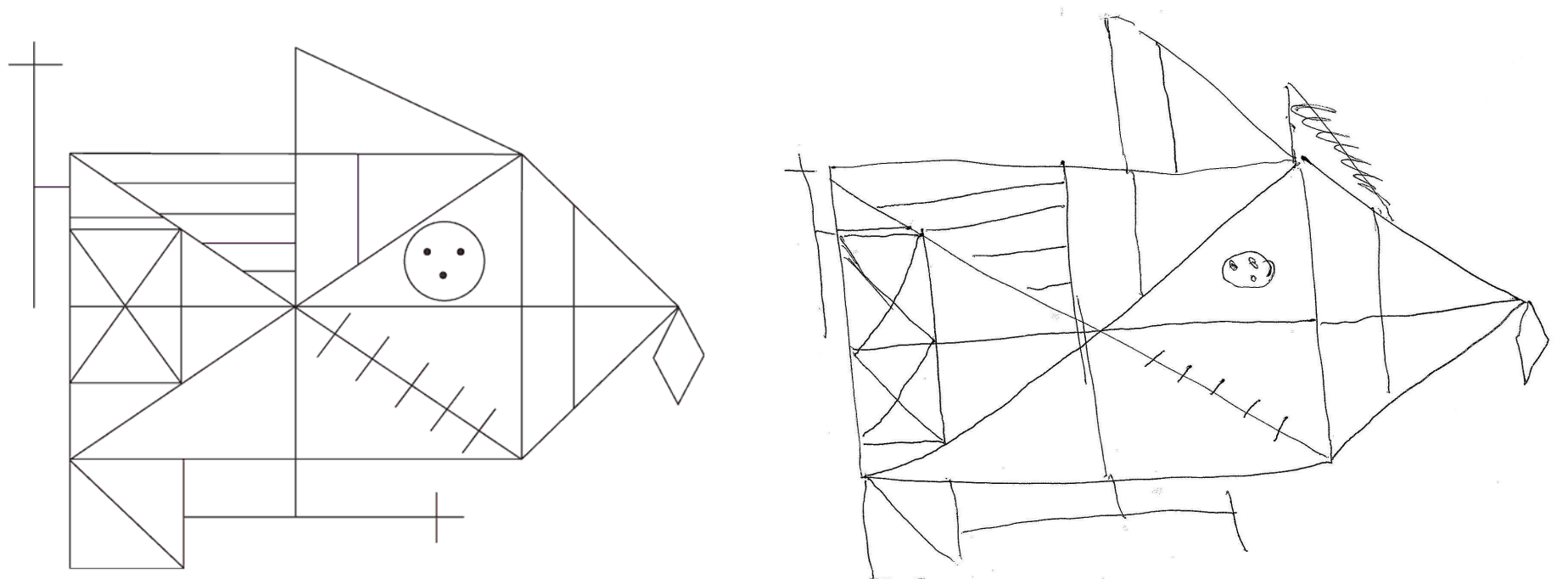


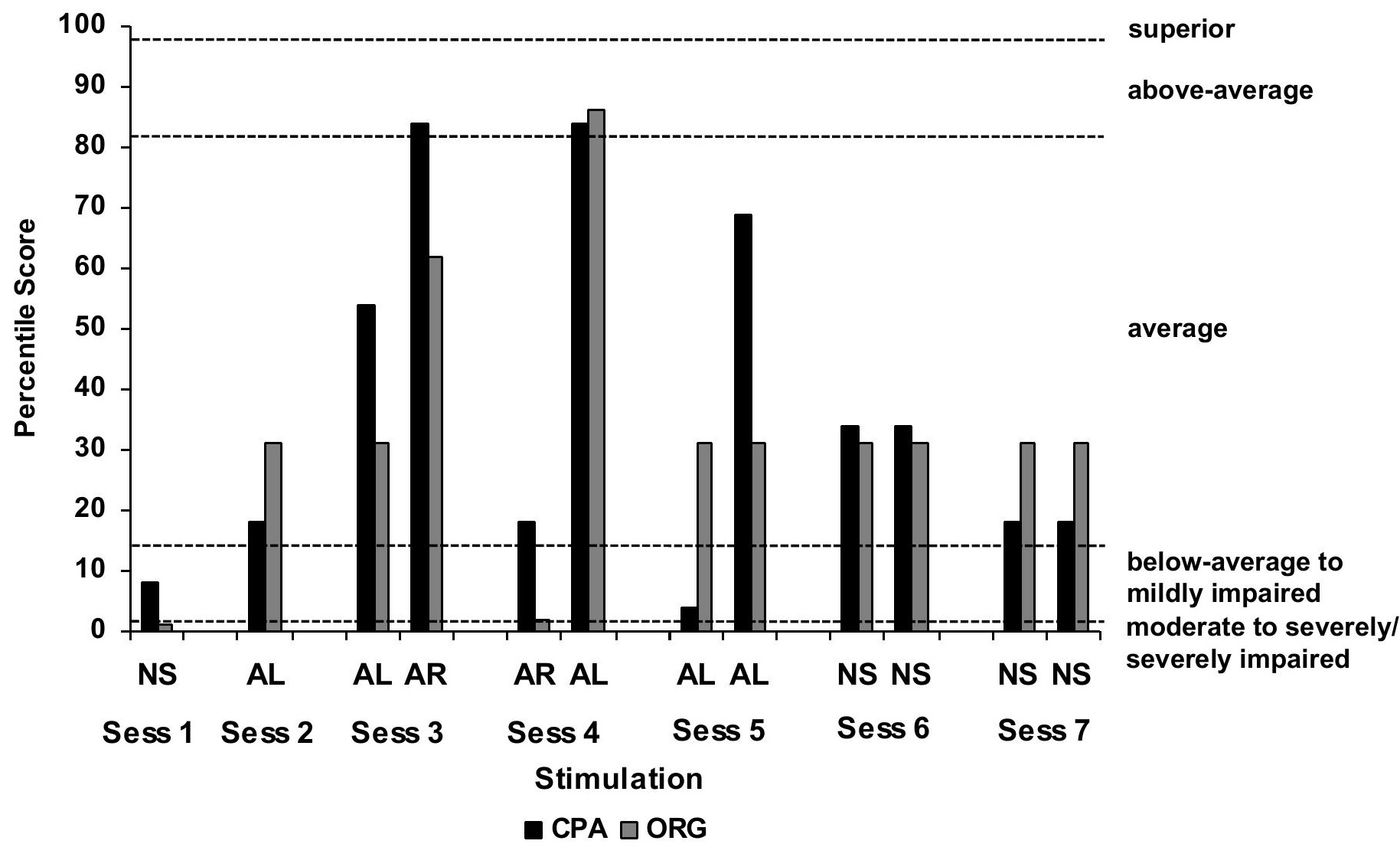

Figure 4 


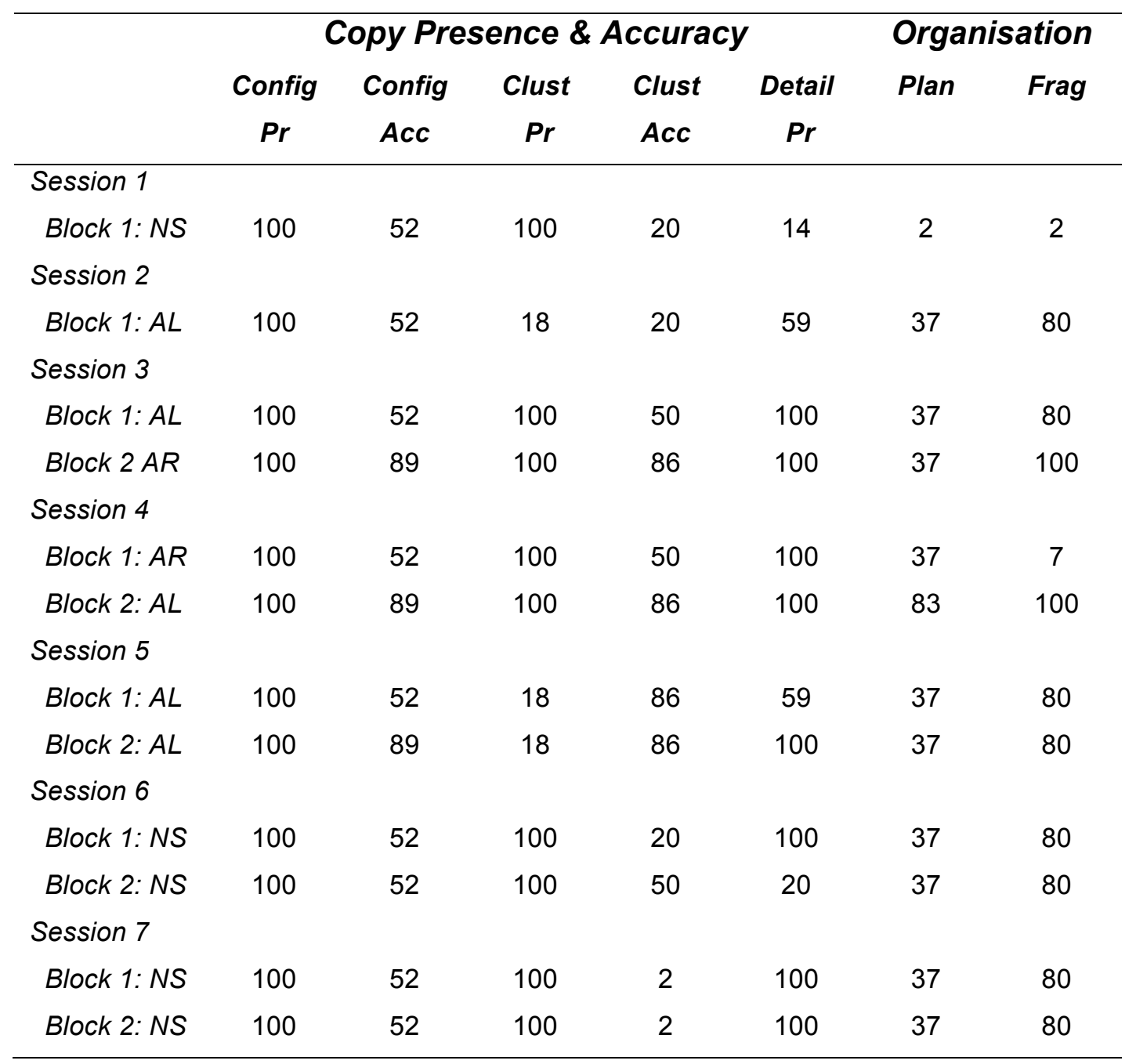

\section{Table 1}

Percentile scores for each of the qualitative scales that comprise the Copy Presence and Accuracy (CPA) and Organisation summary scores. A score of 100 would indicate that all matched controls in the standardised sample received a score at or below the patient's score, while a score of 0 would indicate that all controls scored at or above the patient's score. Key to qualitative scales: Config $=$ configural, Clust $=$ cluster, $\operatorname{Pr}=$ presence, Acc $=$ accuracy, Plan $=$ planning, Frag $=$ Fragmentation. Other abbreviations: $\mathrm{AR}=$ anode right, $\mathrm{AL}=$ anode left, NS = no stimulation (sham). 